\title{
Photoacoustic imaging of tumor targeting with riboflavin-functionalized theranostic nanocarriers
}

\author{
This article was published in the following Dove Press journal: \\ International Journal of Nanomedicine \\ 18 May 2017 \\ Number of times this article has been viewed
}

\author{
Nataliia Beztsinna ${ }^{1, *}$ \\ Yoanna Tsvetkova ${ }^{2, *}$ \\ Jithin Jose 3 \\ Boutayna Rhourri-Frih' \\ Wa'el Al Rawashdeh² \\ Twan Lammers ${ }^{2}$ \\ Fabian Kiessling ${ }^{2}$ \\ Isabelle Bestel' \\ 'Institute of Chemistry \& Biology \\ of Membranes \& Nanoobjects \\ (CBMN), UMR 5248, University of \\ Bordeaux, Pessac, France; ${ }^{2}$ Institute \\ for Experimental Molecular \\ Imaging, RWTH Aachen University \\ Clinic, Aachen, Germany; ${ }^{3}$ Fujifilm \\ VisualSonics BV, Amsterdam, the \\ Netherlands \\ *These authors contributed equally \\ to this work
}

\begin{abstract}
Photoacoustic imaging is an emerging method in the molecular imaging field, providing high spatiotemporal resolution and sufficient imaging depths for many clinical applications. Therefore, the aim of this study was to use photoacoustic imaging as a tool to evaluate a riboflavin (RF)-based targeted nanoplatform. RF is internalized by the cells through a specific pathway, and its derivatives were recently shown as promising tumor-targeting vectors for the drug delivery systems. Here, the RF amphiphile synthesized from a PEGylated phospholipid was successfully inserted into a long-circulating liposome formulation labeled with the clinically approved photoacoustic contrast agent - indocyanine green (ICG). The obtained liposomes had a diameter of $124 \mathrm{~nm}$ (polydispersity index $=0.17$ ) and had a negative zeta potential of $-26 \mathrm{mV}$. Studies in biological phantoms indicated a stable and concentration-dependent photoacoustic signal (Vevo ${ }^{\circledR}$ LAZR) of the ICG-containing RF-functionalized liposomes. In A431 cells, a high uptake of RF-functionalized liposomes was found and could be blocked competitively. First, studies in mice revealed $\sim 3$ times higher photoacoustic signal in subcutaneous A431 tumor xenografts $(P<0.05)$ after injection of RF-functionalized liposomes compared to control particles. In this context, the application of a spectral unmixing protocol confirmed the initial quantitative data and improved the localization of liposomes in the tumor. In conclusion, the synthesized RF amphiphile leads to efficient liposomal tumor targeting and can be favorably detected by photoacoustic imaging with a perspective of theranostic applications.
\end{abstract}

Keywords: photoacoustic imaging, long-circulating liposomes, active targeting, riboflavin, indocyanine green

\section{Introduction}

The photoacoustic (PA) effect, discovered by Alexander Graham Bell in the 19th century, found its application in biomedical imaging only 2 decades ago. ${ }^{1}$ It is based on the ability of certain molecules to generate thermoelastic expansion upon irradiation with short-pulsed laser beams. During this process, the energy of light is converted into acoustic signals. As a consequence, PA imaging combines high spatial resolution of ultrasound (US) and high contrast of optical imaging systems. Moreover, PA imaging allows to gather functional and molecular information in real time; it does not employ ionizing radiation and works at clinically relevant imaging depths. ${ }^{1,2}$ Lately, lots of efforts were put by the scientific community for the development of various biomedical PA imaging applications. Among them are the studies focused on intrinsic PA contrast (hemoglobin and melanin) such as blood vessel growth and antiangiogenic response, microcirculation pathology, biomarkers, brain biology, and drug screening response., ${ }^{2,3}$ However, the use of PA as an imaging modality for nanomedicine evaluation and image-guided drug delivery is just emerging. Few recent studies reported the use of PA imaging to monitor the accumulation of drug carriers in the tumor ${ }^{1,4,5}$ as well as 
for the assessment of important parameters such as tumor oxygenation, total hemoglobin concentration, and regions of hypoxia. ${ }^{6}$ Most of the previously used diagnostic PA agents were non-targeted. Adding a specific ligand to a PA contrast agent will allow selective cell binding and provide additional information about molecular targets' expression in the tumor neovasculature or the extravascular compartment. ${ }^{5,7,8}$

The contrast in PA imaging is achieved with endogenous (hemoglobin, melanin, DNA, RNA, etc) or exogenous absorbers (gold nanoparticles or organic near-infrared [NIR] dyes). Among the latter, indocyanine green (ICG), a clinically approved NIR dye, is one of the most exploited exogenous absorbers so far..$^{4,9-11}$ It has an absorption maximum of $\sim 780-800 \mathrm{~nm}$ (depending on the environment) and fluoresces in the NIR window. In blood, free ICG binds to plasma proteins and is rapidly excreted. It is also unstable in buffer solutions and has a nonlinear fluorescence quantum yield versus concentration. ${ }^{12,13}$ The encapsulation of ICG in liposomes showed a significant improvement of its circulation lifetime, stability, and optical properties in vivo. ${ }^{4,7-14-16}$ Thus, ICG-loaded liposomes are a promising platform for the functionalization with targeting agents to improve their tumor binding, retention, and cellular uptake and for future conception of theranostic nanomedicines.

Among the existing tumor-targeting agents, small molecules represent an attractive alternative to more expensive and complex moieties such as antibodies, aptamers, and peptides. In this context, riboflavin (RF)-based targeting represents a promising approach for cell labeling, ${ }^{17,18}$ tumor contrast enhancement, ${ }^{19}$ and cell-specific drug delivery. ${ }^{20-22}$ Due to its involvement in cell growth and development, RF is of high demand in metabolically active cells such as various malignant (eg, breast, prostate cancer, and hepatocellular carcinoma) and rapidly growing endothelial cells. ${ }^{23-26}$ Thus, a major argument for using RF as a ligand for targeted drug delivery is that it targets both cancerous cells and the tumor stroma including the angiogenic endothelium. ${ }^{19,27,28}$ This feature was demonstrated in several preclinical studies with a variety of used carriers - from iron oxide nanoparticles to polymers. ${ }^{17,18,22,27}$ In our previous study, we validated the efficacy of cancer and endothelial cell targeting in vitro and in vivo by RF amphiphiles inserted into a liposome formulation. ${ }^{28}$ However, the applied combined microcomputed tomography/ fluorescence molecular tomography imaging platform used for these studies has a limited translational potential. Therefore, in this study, PA imaging is introduced for the evaluation of a RF-functionalized tumor-targeted nanoplatform, opening new perspectives to study the accumulation of this nanomedicine in large animals and in a clinical environment.

\section{Synthesis and formulation}

The DSPE-PEG-RF amphiphile was prepared in a single step from commercially available DSPE-mPEG2000-succinyl as described previously. ${ }^{28}$ One of the main features of this conjugate is that both components RF and DSPE-PEG are clinically approved and are bound with a biodegradable ester linkage. This might facilitate the RF amphiphile translation to the clinics.

DSPE-PEG-RF was inserted into a well-known lipid formulation - DPPC:cholesterol:DSPE-PEG2000 with a 65:30:5 molar ratio. Such lipid composition was reported ${ }^{29-31}$ as stable and is similar to commercially available liposomal products like Doxil ${ }^{\circledR}$, Lipo-dox ${ }^{\circledR},{ }^{31}$ and liposome oxaliplatin. ${ }^{32}$ Liposomes were prepared with a standard film hydration method, and DSPE-PEG-RF was dissolved in chloroform and mixed with other lipids during film preparation. Our previous study demonstrated that this strategy allows proper incorporation of the RF amphiphiles in the liposome bilayer. ${ }^{28}$ The formulation containing DSPE-PEG-RF is further referred to as targeted liposomes (Figure 1). The liposomes with no RF amphiphile were used as controls. The physicochemical characterization of the prepared formulations is presented in Figure 1.

For liposome labeling, the clinically approved chromophore and PA contrast agent ICG was used. An appropriate amount of ICG was dissolved in HEPES buffer and used for lipid film hydration. ICG is mainly a water-soluble fluorophore, but in liposomes it is generally situated in the lipid bilayer due to its amphiphilic nature. This could be visualized by the changes in ICG absorption spectrum (Figure S1) as the molecule is highly environment sensitive.

The presence of both RF and ICG in the formulation was confirmed spectroscopically. At the fluorescence emission spectra (Figure 1), one could observe a peak at $530 \mathrm{~nm}$ characteristic for RF in targeted liposomes and the beginning of an ICG emission in the NIR region in both targeted and control formulations. The exact amount of ICG and RF in the formulations was calculated with the help of calibration curves $y=59087 x\left(R^{2}=0.9939\right)$ for $\mathrm{RF}$ and $y=21604 x\left(R^{2}=0.9591\right)$ for $\mathrm{ICG}$, where $y$ represents fluorescence intensity for RF and absorbance at $780 \mathrm{~nm}$ for ICG and $x$ represents concentration in $\mathrm{mM}$ (Figure S1). The control liposomes contained a slightly lower amount of encapsulated ICG than the targeted liposomes (92 and $110 \mu \mathrm{M}$, respectively).

The average liposome size for both formulations was $\sim 120 \mathrm{~nm}$ and the acceptable polydispersity index (PDI) was $\sim 0.17$. As discussed in the previous study, ${ }^{28}$ even after purification by high-pressure liquid chromatography (HPLC), 


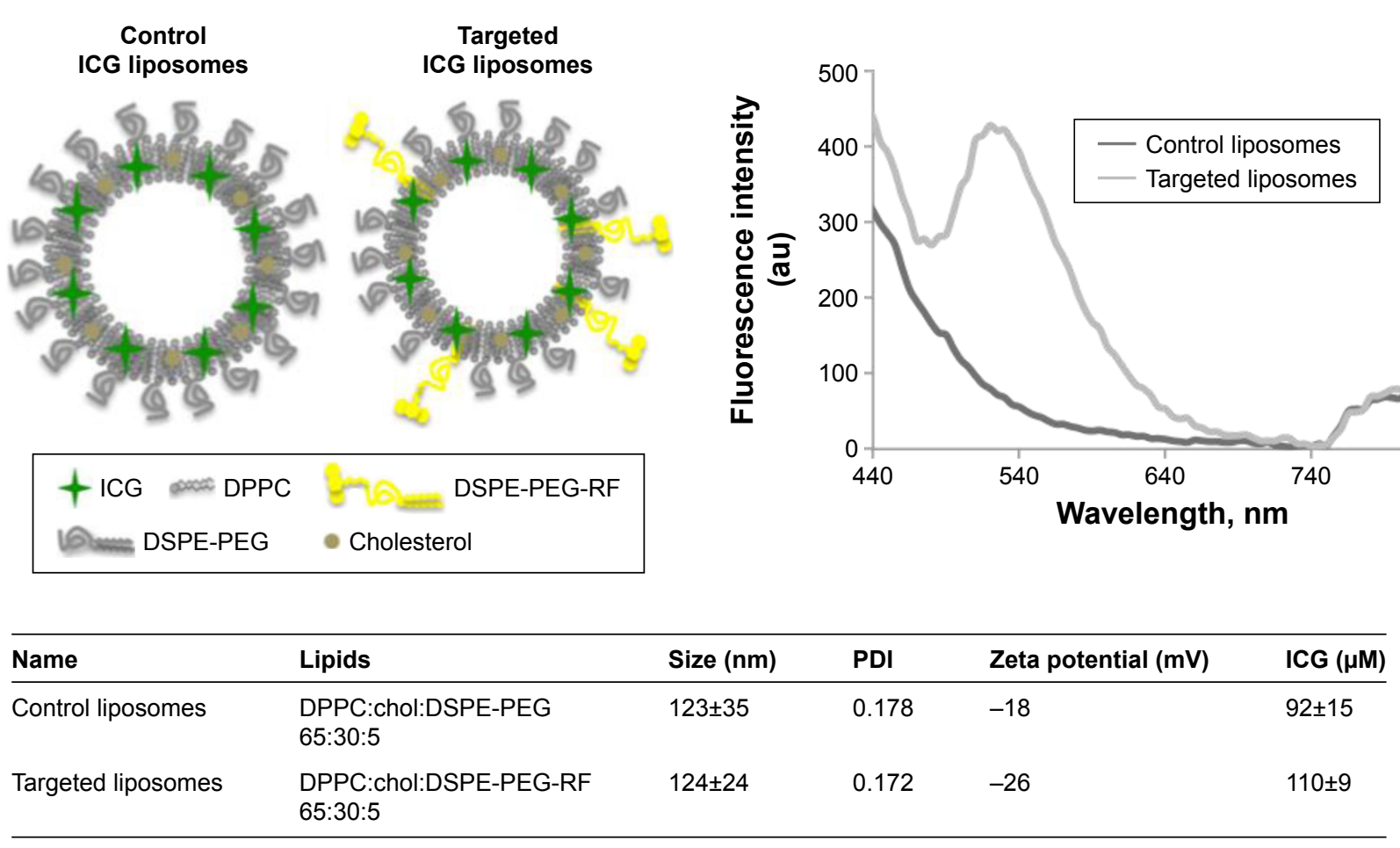

Figure I Scheme of control and targeted ICG liposomes (top left); fluorescence spectra of control and targeted liposomes (top right); and major characteristics of prepared formulations (bottom).

Abbreviations: ICG, indocyanine green; PDI, polydispersity index; DPPC, dipalmitoyl phosphocholine; DSPE-PEG, distearoyl phrosphoethanolamine-polyethylene glycol; DSPE-PEG-RF, distearoyl phrosphoethanolamine-polyethylene glycol-riboflavin.

a mixture of unreacted DSPE-PEG-COO- ${ }^{-}$and the desired conjugate DSPE-PEG-RF was detected. The additional negative charges introduced by the carboxylic acid moiety might explain the slightly lower zeta potential of the targeted liposomes $(-26 \mathrm{mV})$ compared to that of the control formulation $(-18 \mathrm{mV})$.

The liposome stability was monitored in 50\% fetal bovine serum (FBS) at $37^{\circ} \mathrm{C}$ over time. The results presented in Figure $\mathrm{S} 1$ indicate no significant changes in ICG absorption after incubation with FBS up to 48 hours for both control and targeted formulations. The mean hydrodynamic diameter, PDI, and zeta potential of liposomes after incubation with FBS were also verified. For the control liposomes, the average hydrodynamic size was slightly higher $(+5 \mathrm{~nm})$ and PDI increased by 2.7 times. The zeta potential of control liposomes became twice more negative - from -18 to $-36 \mathrm{mV}$. As for the targeted formulation, the mean hydrodynamic size increased slightly, the zeta potential did not change, and PDI increased by 3.7 times. It should be noted that a targeting agent on the surface of long-circulating liposomes is always a risk that might lead to the reduced circulation half-life and, as a consequence, compromised passive accumulation in the tumor via EPR effect. ${ }^{33,34}$ However, in the previous study, ${ }^{28}$ the RF-functionalized liposomes did not show a reduced circulation time compared to the control formulation.
In summary, DSPE-PEG-RF was successfully inserted into a liposomal formulation with an encapsulated ICG. Compared to the control liposomes, the amphiphile induced only slight changes in the zeta potential toward more negative values. This might on the one hand enhance the colloidal stability of the liposomes but, on the other hand, decrease their cellular uptake due to steric repulsions. ${ }^{35}$ Nevertheless, in the case of active targeting, such a negative charge might be even beneficial as it could potentially decrease nonspecific interactions between liposomes and cells. The ICG absorbance signal in liposomes remained stable even after 48-hour incubation in 50\% FBS, and the liposome size distribution was not dramatically affected as well.

\section{In vitro evaluation}

The targeting efficiency of the DSPE-PEG-RF derivative was evaluated in vitro in A431 cells. Although, ICG is clinically applied, it is difficult to handle under in vitro conditions because of its high fluorescence emission wavelength $(>800 \mathrm{~nm})$, which requires a special microscopy equipment. For this reason, rhodamine B (RhB) was encapsulated in the control and targeted liposomes to perform uptake studies in A431 cells. Non-labeled targeted liposomes were additionally prepared for competitive binding studies. Liposomes were prepared in the same way 
as the ICG-containing formulations and exhibited similar characteristics (Table S1).

The A431 cells were incubated with control and targeted liposomes ( 5 pmol of encapsulated $\mathrm{RhB}$ ) for 30 minutes at $37^{\circ} \mathrm{C}$. The uptake was monitored by fluorescence microscopy and quantified by the RhB fluorescence signal (ex. 554, em. 605). The DSPE-PEG-RF-containing liposomes showed a 5.9 times higher $(95 \pm 18 \mathrm{au})$ uptake compared to the control liposomes (16 \pm 2 au, $P<0.001)$ (Figure 2). The competitive binding studies, where cells were preincubated with 20 -fold excess of non-labeled liposomes, showed a significant decrease $(26 \%, P<0.001)$ in the targeted liposome uptake. These data suggest that the higher internalization of DSPEPEG-RF liposomes than of control ones is mediated via RF transporters, which is in agreement with previous in vitro studies on RF-transporter targeting. ${ }^{18,22,28}$

\section{Phantom experiments}

Prior to the application of our PA contrast agent in vivo, its performance was tested in biological phantoms. Here, polymeric blood vessel-mimicking tubes were used to evaluate the PA detection limit upon liposome dilution and to register the PA spectrum. Furthermore, a chicken muscle tissue phantom was used to mimic a biological environment and to determine the signal sensitivity at various depths.

The targeted ICG-containing liposome stock solution was diluted to obtain a concentration range from 2 to 2,000 pmol in $35 \mu \mathrm{L}$. These solutions were injected into blood vessel-mimicking tubes, and the tubes were subjected to PAI in combination with conventional US. The intensity of the PA signal from liposomes in the tubes was acquired in the range from 680 to $970 \mathrm{~nm}$ (Figure 3A). The maximum PA intensity of ICG in liposomes was observed after laser irradiation at $806 \mathrm{~nm}$ and shifted upon dilution to $800 \mathrm{~nm}$. The PA signal displayed in red in Figure 3B was overlaid with the US signal from the tubes (grey). PA average intensity (IPA) was calculated in the selected regions of interest (ROIs) and then plotted against ICG amount in pmol. The data show that liposomes were still detectable at a concentration of 2 pmol. The concentration dependence of the PA signal was logarithmic, expressed by the equation $y=0.2872 \ln (x)+0.3643$, where $y$ represents the PA signal intensity and $x$ represents the quantity of ICG liposomes in pmol (Figure S1).

To determine the maximum depth of signal penetration, $2 \mathrm{nmol}$ of liposomes in $50 \mu \mathrm{L}$ were placed at different depths in a chicken muscle phantom. The PA signals were again overlaid on the US images. An exponential decline in the PA signal intensity with the increase of injection depth $(\mathrm{d}, \mathrm{mm})$ was observed, which is in accordance with the previously published data from Repenko et al. ${ }^{36}$ The ICG liposome signal was clearly resolved till the depth of $10 \mathrm{~mm}$, which is the relevant depth for subcutaneous tumor xenografts in mice. ${ }^{37}$ Such a signal depth is also appropriate for several clinical applications such as monitoring of drug delivery to superficial skin tumors ${ }^{38}$ as well as endoscopic detection of gastrointestinal tumors, ${ }^{8}$ but certainly is not the limit that can be achieved in vivo.

\section{In vivo evaluation}

The accumulation of control and targeted ICG-labeled liposomes was evaluated in nude female mice bearing epidermoid carcinoma (A431) xenografts. The animals were divided into two groups with $n=5$ in each and received single intravenous injection of either control or targeted formulation (100 $\mu \mathrm{L}$ of liposome solution with the total amount of $6 \mathrm{nmol}$ of ICG per mouse). All tumors were imaged with the PA Vevo ${ }^{\circledR}$ LAZR system (FUJIFILM VisualSonics, Amsterdam, the Netherlands) at $800 \mathrm{~nm}$ before injection and 15 minutes and 4 hours after injection. The tumors were scanned in three dimension (3D) with the help of an automated motor system.
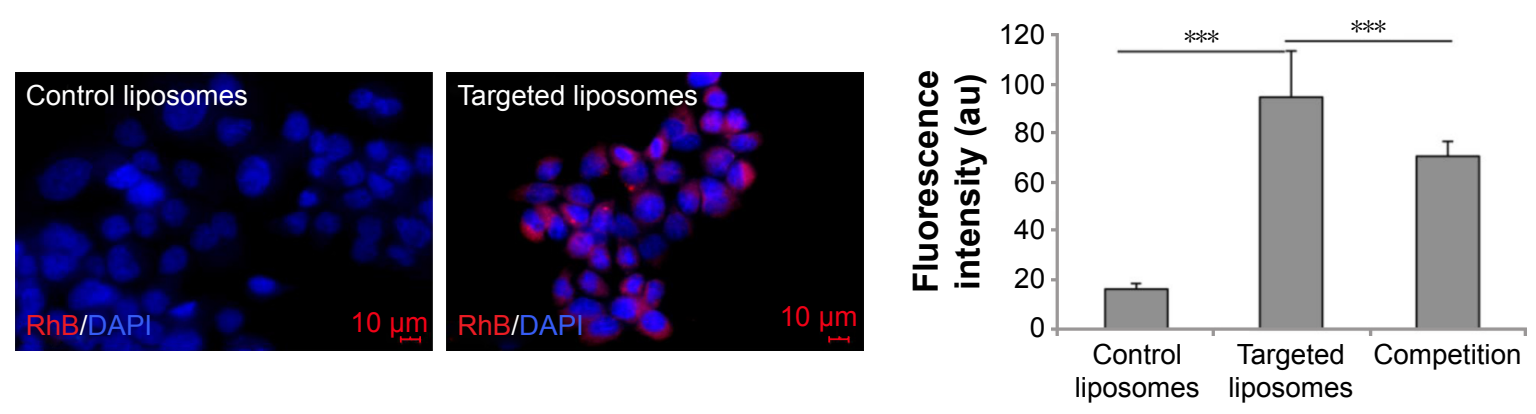

Figure 2 Fluorescence microscopy images of control and targeted liposomes' uptake in A43I cells (left) and uptake quantification (right).

Notes: RhB (red) and cell nuclei marked with DAPI (blue); results are presented as mean from three individual experiments with $\mathrm{n}=10$ images for each condition. $* * * P<0.001$.

Abbreviations: RhB, rhodamine B; DAPI, 4',6-diamidino-2-phenylindole. 

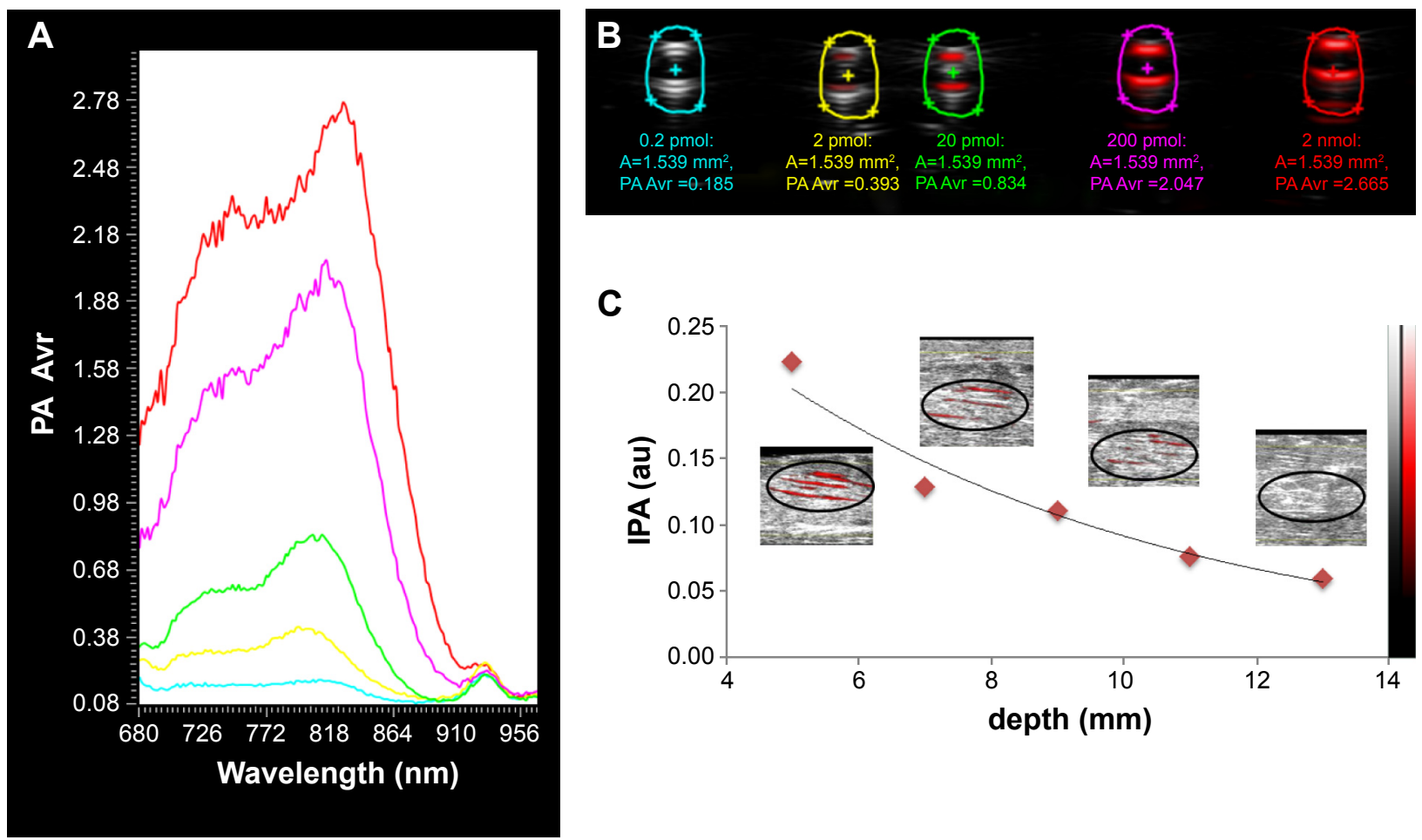

C

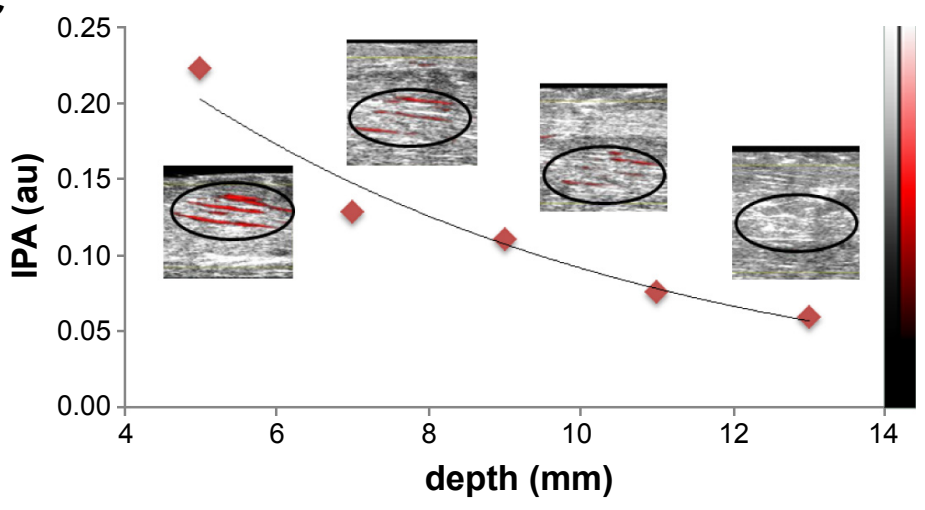

Figure 3 (A) PA spectra of ICG liposomes at various concentrations, colors corresponding to ROls from (B). (B) Overlay of US (grey) and PA (red) images from ICG liposomes at various concentrations. (C) Depth dependence of IPA signal from ICG liposomes in chicken breast phantom.

Abbreviations: PA, photoacoustic; ICG, indocyanine green; ROI, region of interest; US, ultrasound; Avr, average; IPA, PA average intensity.

For each time point, the tumors were reconstructed in 3D (Figure S2), and the relative increase in the mean PA signal per tumor volume (related to the value before injection) was calculated (photoacoustic signal $[\mathrm{PS}] \%$ ) using the $\mathrm{Vevo}^{\circledR}$ LAB software (Figure 4).
Due to the presence of a specific ligand, the early kinetics of a targeted nanosystem within the tumor are expected to differ from a non-targeted one. ${ }^{7,39}$ At later time points, the conventional PEGylated liposomes reach their peak in tumor accumulation via the EPR effect, and the expected
A

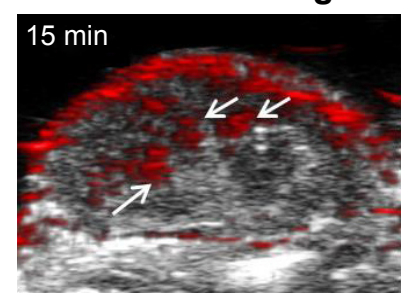

Targeted liposomes

\section{Control liposomes}

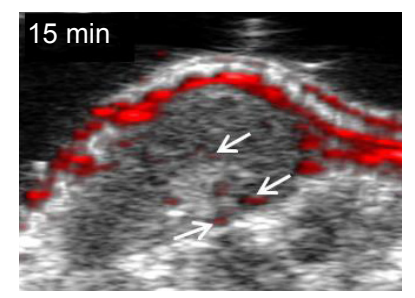

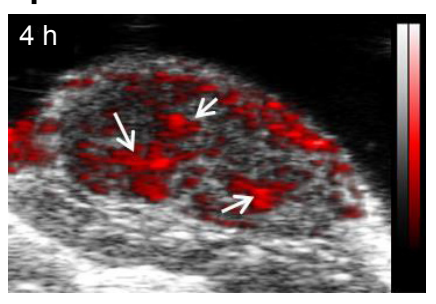

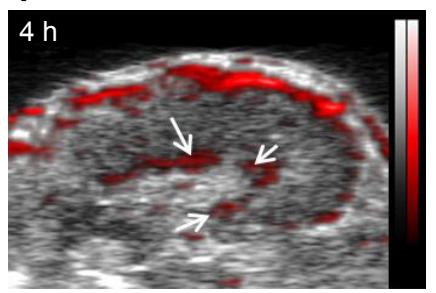

B

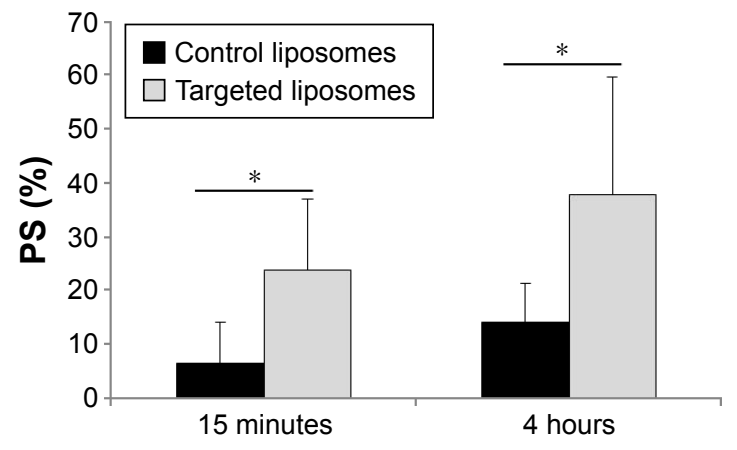

Figure 4 (A) Examples of two-dimensional tumor slices at different time points after injection of targeted or control liposomes. (B) Normalized PS\% signal intensity in three-dimensional tumor volume after liposome injection.

Notes: US signal (black and white) overlapped with PA signal (red). Arrows indicate signal accumulation in the tumor. Data are presented as mean \pm standard deviation from $n=5$ mice/group. $* P=0.05$.

Abbreviations: US, ultrasound; PA, photoacoustic; h, hours; min, minutes. 
A Control liposomes

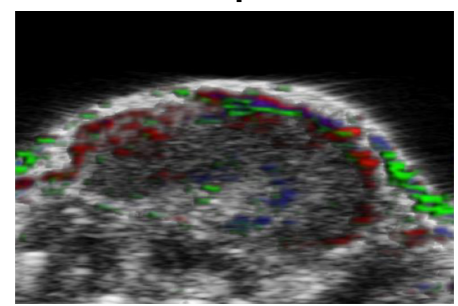

Targeted liposomes

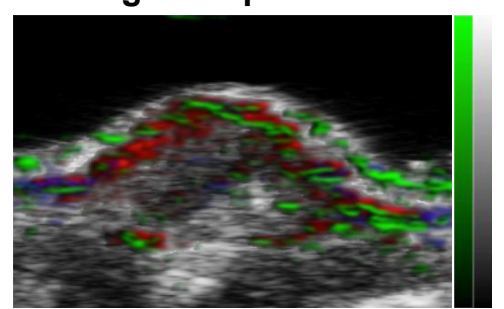

B

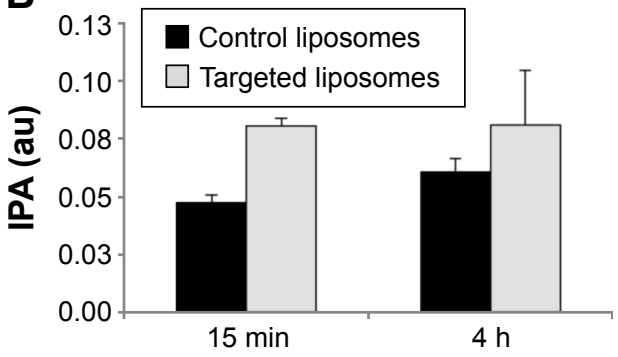

Figure 5 (A) Merge of US (black and white) and PA unmixed images of 2D tumor slices 4 hours after injection of control or targeted liposomes. (B) Quantification of unmixed IPA from 2D tumor slices.

Notes: Green - ICG signal from liposomes; red - OxyHemo; and blue - deOxyHemo. Data are presented as mean \pm standard deviation from $\mathrm{n}=2 \mathrm{mice} / \mathrm{group}$.

Abbreviations: US, ultrasound; PA, photoacoustic; 2D, two-dimensional; ICG, indocyanine green; IPA, PA average intensity; h, hours; min, minutes.

difference between the targeted and the non-targeted becomes less obvious. ${ }^{39}$ Our previous studies already demonstrated the overall capability of RF-functionalized nanosystems for multicompartment tumor targeting ${ }^{18,27,28}$ as well as the prolonged circulation of DSPE-PEG-RF-functionalized liposomes. Therefore, in this study, the focus was on the qualitative and quantitative differences in targeted and control liposome accumulation at early time points, which can be clearly assigned to (endothelial) targeting effects.

Indeed, at 15 minutes and 4 hours after injection, a significant increase in PS\% was observed in the targeted group compared to the control group (Figure 4B). The average increase in PS\% for the targeted group was $24 \% \pm 13 \%$ after 15 minutes and $38 \% \pm 22 \%$ after 4 hours. In contrast, the control group showed only a moderate $7 \% \pm 6 \%$ increase after 15 minutes and reached $14 \% \pm 7 \%$ after 4 hours. Consequently, the accumulation of targeted liposomes was 3.4 times higher than that of control liposomes already after 15 minutes and 2.6 times higher after 4 hours $(P<0.05)$. The observed high PS\% signal variability might be due to the differences in blood perfusion among xenografts. Similarly with a previous study from Song et al, ${ }^{11}$ both liposomal formulations were found heterogeneously distributed within the tumor (Figure 4) with the preferential localization next to major tumor-supplying blood vessels. The higher accumulation of RF-functionalized liposomes is assumed to be associated with endothelial cell binding. ${ }^{19,27}$ Even though the addition of a targeting ligand on the surface of long-circulating liposomes might carry the risk of reducing nanomedicines' blood half-life or tumor extravasation, it is still a valuable alternative for the intracellular delivery of fragile and highly active biomolecules (eg, nucleic acids or proteins). ${ }^{34}$ In this context, the endothelial cell targeting is a highly promising approach as it allows fast binding and internalization and does not require deep tumor extravasation.
To reduce the influences of the intrinsic blood signal on the measurements, spectral unmixing ${ }^{40}$ was applied to some of the studied animals ( $n=2 /$ group). The protocol is able to distinguish the PA spectrum of a particular compound from the main blood absorbers - oxygenated (OxyHemo) and deoxygenated (deOxyHemo) forms of hemoglobin. ${ }^{4}$ For this purpose, the PA data were registered at various wavelengths $-700,750,800,850$, and $900 \mathrm{~nm}$ - in two-dimensional (2D) tumor slices. Then, the acquired data were automatically unmixed using the Vevo LAB software considering the PA spectrum of ICG-labeled liposomes (Figure 3 ) and the blood components (existing as a preset in the software). Figure $5 \mathrm{~A}$ demonstrates an example of an unmixed 2D tumor slice 4 hours after the injection of control and targeted liposomes. Similar to the non-unmixed PA image (Figure 4A), the ICG signal was non-homogeneously distributed within the tumor and localized next to tumor blood vessels and surrounding areas. The calculated PA intensities (IPA) from tumor ROIs in unmixed images (Figure S3) after the injection of either targeted or control liposomes are presented in Figure 5B. The differences in the IPA profiles between both formulations were in line with the PS\% signal increase discussed earlier, where the targeted liposomes displayed a higher accumulation than the control formulation. In contrast, the averaged PA signal from oxygenated and deoxygenated hemoglobin was not significantly different between groups (Figure S4), indicating comparable blood supply in the tumors of both groups.

\section{Conclusion}

In summary, the described RF-functionalized nanocarrier was stable and exhibited a good PA signal. Compared to the control particles, it showed a higher cell uptake in vitro and enhanced tumor accumulation in squamous cell carcinoma 
xenografts in vivo indicating the suitability of RF as a targeting ligand for cancer nanomedicines. Moreover, this study demonstrated the ability of PA imaging to gather quantitative information about nanomedicines' accumulation in tumors. Thus, PA imaging is a promising tool to measure the tumor accumulation of nanomedicines together with functional information about tumor vascularization and oxygenation.

\section{Experimental section}

Reagents and solvents were purchased from Sigma Aldrich (Taufkirchen, Germany), Glen Research (Sterling, VA, USA), or Alfa Aesar (Thermo Fisher Scientific, Waltham, MA, USA) and were used without further purification. Phospholipids were purchased from Bachem AG (Bubendorf, Switzerland) or Avanti Polar Lipids (Alabaster, AL, USA). Fluorescent dyes were purchased from Sigma Aldrich (Germany). Cell culture media and additives were from Gibco ${ }^{\circledR}$ (Thermo Fisher Scientific, Waltham, MA, USA).

For the mass spectrometry analysis, an Ultraflex III MALDI-TOF-TOF instrument (Bruker Daltonics $\mathrm{GmbH}$, Bremen, Germany) equipped with a Smartbeam 2 laser with a repetition rate up to $200 \mathrm{~Hz}$ (500 laser shots) was used. The obtained mass spectrum was analyzed with Flex-analysis version 4.0 software (Bruker Daltonics $\mathrm{GmbH}$ ).

The HPLC separation and analysis were performed with Waters $^{\circledR}$ Alliance 2695 (Waters ${ }^{\circledR}$ Corporation, Milford, MA, USA) apparatus equipped with the ultraviolet (UV) detector Waters $^{\circledR} 486$ (Waters Corporation) and analyzed with Millennium 32 software (Waters Corporation). The reverse phase analytical C8 column (Macherey-Nagel, GmbH \& Co, KG, Hoerdt, France) was used for all separations.

\section{Synthesis of DSPE-PEG-RF}

DSPE-PEG-RF was prepared as described previously. ${ }^{28}$ Briefly, 10 mg of DSPE-PEG2000-succinyl (1,2-distearoyl-snglycero-3-phosphoethanolamine- $N$-[succinyl(polyethylene glycol)-2000] [ammonium salt]; $0.34 \mu \mathrm{mol}$ ) was mixed with $2.5 \mathrm{mg}$ RF (20 eq, $6.8 \mu \mathrm{mol}$ ) dissolved in anhydrous dimethylformamide. The catalytic amount of 4-dimethylaminopyridine and stoichiometric amount of $N, N^{\prime}$-dicyclohexylcarbodiimide were added, and the reaction mixture was left under nitrogen stream and continuous magnetic stirring for 48 hours. After the completion of reaction, the solvent was evaporated by lyophilization (Dura-Dry ${ }^{\mathrm{TM} M P}$, FTS Systems ${ }^{\mathrm{TM}}$, Warminster, PA, USA), and the crude product was dissolved in $\mathrm{H}_{2} \mathrm{O}$ / ACN 50:50 mixture and purified by HPLC on the reverse phase analytical C8 column (Macherey-Nagel, GmbH \& $\mathrm{Co}, \mathrm{KG}$ ) (solvent $\mathrm{A}-\mathrm{H}_{2} \mathrm{O} / \mathrm{ACN} / \mathrm{TFA}$ 50:50:0.1; solvent
$\mathrm{B}-\mathrm{H}_{2} \mathrm{O} / \mathrm{ACN} / \mathrm{TFA} 50: 50: 0.1$ ), with the gradient 0\%-100\% $\mathrm{B}$ for 25 minutes and then $100 \%-0 \%$ B for 5 minutes. The UV detector was set at $265 \mathrm{~nm}$. Then, the gathered fractions were lyophilized and analyzed with MALDI-TOF-TOF mass spectrometry (Bruker Daltonics GmbH) (Figure S5).

Mass observed: 3,288 uma; 2,438 uma.

\section{Liposome preparation and characterization}

The liposomes were prepared with the standard film hydration method. The final concentration of lipids was $20 \mathrm{mM}$. Briefly, the lipids were dissolved in chloroform, mixed in appropriate proportions in the round-bottom flask, and the solvent was evaporated under vacuum. The flask was then put into a lyophilisator (Dura-Dry ${ }^{\text {TMMP, FTS Systems }}{ }^{\text {TM }}$ ) overnight to remove any residual solvent traces. The film was hydrated with $1 \mathrm{~mL}$ of $10 \mathrm{mM}$ HEPES buffer ( $\mathrm{pH}=7.4$ ) with $9.25 \%$ sucrose-containing $0.5 \mathrm{mg} / \mathrm{mL}$ ICG (Sigma Aldrich) or RhB (Sigma Aldrich) for 1 hour at $60^{\circ} \mathrm{C}$. The hydrated films were then subjected to five freeze-thaw cycles with the help of liquid nitrogen and a water bath (same temperature than for hydration). The obtained multilamellar vesicles were then extruded 11 times through 800, 400, 200, and finally $100 \mathrm{~nm}$ polycarbonate membranes (Avanti Polar Lipids). To separate the liposomes from the free dye, they were purified by sizeexclusion chromatography with Sephadex G75 resin (Sigma Aldrich). The liposome solutions were stored at $4{ }^{\circ} \mathrm{C}$.

The size, distribution, and zeta potential measurements of control and targeted liposomes were performed in triplicate by dynamic light scattering (DLS) using Zetasizer Nano ZS (Malvern Instruments, Malvern, UK) at $25^{\circ} \mathrm{C}$. Calculations of size distribution based on light scattering intensity were performed by the Zetasizer Nano software from the correlation functions using the general purpose algorithm (Malvern Instruments). The liposomal stock dispersions were diluted by a factor of 15 with $10 \mathrm{mM}$ HEPES buffer before measurements performed by DLS. The size distribution data are presented in Figure S6.

The liposome fluorescence intensity and absorbance were measured with Tecan Infinite 200 Pro (Tecan Trading AG, Männedorf, Switzerland) and analyzed with Tecan i-control, v. 1.7.1.12, software (Tecan Trading AG). The fluorescence calibration curves prepared for RF (ex. $450 \mathrm{~nm}$, em. $530 \mathrm{~nm}$ ) and $\mathrm{RhB}$ (ex. $554 \mathrm{~nm}$, em. $583 \mathrm{~nm}$ ) were described with the following equations: $y=59087 x\left(R^{2}=0.9939\right)$ and $y=17888 x$ $\left(R^{2}=0.98918\right)$, respectively, where $y$ represents the fluorescence intensity and $x$ represents the concentration in $\mu \mathrm{M}$. ICG was quantified by its absorbance at $780 \mathrm{~nm}$ after liposome 
dilution with $10 \%$ dimethyl sulfoxide. The calibration curve was characterized by the following equation: $y=21604 x$ $\left(R^{2}=0.9591\right)$, where $y$ represents the ICG absorbance at $780 \mathrm{~nm}$ and $x$ represents its concentration in $\mathrm{mM}$.

\section{Cell culture and uptake experiments}

The A431 (human epidermoid carcinoma) cells were maintained at $37^{\circ} \mathrm{C}$, under humidified air with $5 \% \mathrm{CO}_{2}$, in Roswell Park Memorial Institute medium supplemented with 10\% (v/v) FBS and $100 \mathrm{U} / \mathrm{mL}$ of penicillin $\mathrm{G}$ and $100 \mathrm{U} / \mathrm{mL}$ of streptomycin (cell culture media and additives were from Gibco [Thermo Fisher Scientific]). The cells were cultivated in standard $75 \mathrm{~cm}^{2}$ flasks and split each 3-5 days (up to ten passages maximum).

For the uptake studies, the cells in maintenance medium were trypsinized and seeded into 24-well culture plates at optimum confluence. The incubations were conducted at $50 \mathrm{nM}(\mathrm{RhB})$ of control or targeted liposomes in the serumcontaining maintenance medium for 30 minutes. For the competitive binding experiment, the cells were preincubated with 20-fold excess of targeted liposomes without any dyes for 15 minutes and then, without washing, the targeted liposomes were added and incubated for 30 minutes. For the $4^{\circ} \mathrm{C}$ uptake studies, the cells were preincubated in the refrigerator for 30 minutes. After incubation with liposomes, the cells were washed three times in $1 \times$ PBS to remove free liposomes and fixed with $4 \%$ paraformaldehyde. The cell nuclei were stained with 4',6-diamidino-2-phenylindole (DAPI) following the manufacturer's protocol (Life Technologies). The cellular uptake was monitored by fluorescence microscopy with an Axio Imager M2 microscope and a high-resolution AxioCam MRm Rev.3 camera (Zeiss Group, Oberkochen, Germany) under $20 \times$ or $40 \times$ magnifications. Various channels were imaged - DAPI (ex. $365 \mathrm{~nm}$, em. $455 \mathrm{~nm}$ ) to visualize nuclei and DsRed (ex. $554 \mathrm{~nm}$, em. $605 \mathrm{~nm}$ ) to observe liposome uptake trough RhB fluorescence. For each individual well, more than ten images were collected, and the fluorescence intensity was then quantified with the ImageJ software (US National Institutes of Health, Bethesda, MD, USA). All uptake experiments were conducted in triplicates.

\section{PA imaging}

PAI was performed on the Vevo LAZR system equipped with a LZ250 transducer (FUJIFILM VisualSonics, Amsterdam, the Netherlands) as described previously. ${ }^{36}$ The Vevo LAZR system features peak energies of $45 \pm 5 \mathrm{~mJ}$, with a pulse duration of 4-6 ns at adjustable wavelengths between 680 and $970 \mathrm{~nm}$ (2 nm step size). In combination with the PA signal, high-resolution US images were acquired at $21 \mathrm{MHz}$ (central frequency). Both these conditions were maintained for the phantom and in vivo experiments.

\section{Phantom studies}

To investigate the depth detection limit of ICG liposomes, $50 \mu \mathrm{L}$ of the liposome stock solution $(100 \mu \mathrm{M})$ were injected intramuscularly into the chicken muscle tissue at different depths. For all experiments, the imaging parameters were kept constant. For the measurements in blood vessel-mimicking plastic tubing phantoms, a $100 \mu \mathrm{M}$ solution of ICG liposomes was diluted five times by a factor of 10 to obtain the final ICG content of 2,000, 200, 20, 2, and 0.2 pmol. The dilutions were injected into polyethylene plastic tubes, aligned parallel in a custom-built rack and immersed in water (as background). As a control, one of the tubes was filled with the HEPES buffer solution. The immersed tubes were then subjected to simultaneous PA and US imaging providing the PA excitation spectra at various concentrations. At the maximum wavelength, merged US/PA images of the plastic tubes were recorded.

\section{Tumor accumulation studies}

All animal experiments and study protocols followed the guidelines of Gesellschaft für Versuchstierkunde/ Society of Laboratory Animal Science, were approved by local and national German regulatory authorities and by ethical animal welfare committee under the number $\mathrm{AZ}$ - 87-51.04.2010.A278, and complied with the European Convention of Animal Protection laws.

Eight-week-old CD1-nu/nu mice (Charles River Laboratories, Wilmington, DE, USA) weighing $\sim 25 \mathrm{~g}$ were fed with food pellets and water ad libitum. Mice were housed in ventilated cages and placed in a controlled room with adapted conditions. A431 human epidermoid carcinoma cells were cultured as described in the abovementioned section. Mice were injected with A431 $\left(5 \times 10^{6}\right.$ cells/100 $\left.\mu \mathrm{L}\right)$ by subcutaneous inoculation into the dorsolateral right flank. A tumor size of $\sim 5-8 \mathrm{~mm}$ was obtained within 10-14 days for A431 cells. The animals were conditionally inhalation-anesthetized during all experimental operations.

The control and targeted ICG liposomes $(100 \mu \mathrm{L}$ with $6 \mathrm{nmol}$ ICG in each) were injected into the tail vein of $15 \mathrm{CD}-1$ nude female mice bearing A431 tumors with a size of $\sim 5-8 \mathrm{~mm}$ in diameter. The animals were divided into two groups - control and targeted with $\mathrm{n}=5$ in each group. The anesthetized $\left(2.5 \%\right.$ mixture of isoflurane $\left./ \mathrm{O}_{2}\right)$ animal was placed in the Vevo ${ }^{\circledR}$ Imaging Station system, and the tumor was first located 
with a high-resolution US. Then the tumors were scanned in $3 \mathrm{D}$ with the help of an automated motor system. The PA signal was acquired at $800 \mathrm{~nm}$ excitation wavelength together with the US signal. For several animals ( $n=2$ in each group), the PA signal was also acquired at multiple excitation wavelengths in the NanoStepper mode - 700, 750, 800, 850, and $900 \mathrm{~nm}$ - in $2 \mathrm{D}$ tumor slices. All tumors were scanned prior to liposome injection and at 15 minutes and 4 hours after injection. After the last scan, the animals were sacrificed. The tumor segmentation, the PA signal intensity calculations, and spectral unmixing were performed with Vevo LAB 2.2.0 software.

\section{Statistical analysis}

All data are presented as mean \pm standard deviation. Twotailed Student's $t$-test and the variance analysis were used to determine significance among the groups. A $P$-value of $<0.05$ was considered to show significant differences.

\section{Associated content}

All additional experimental data are presented in the Supplementary materials. It is available free of charge via the Internet.

\section{Acknowledgments}

This work was supported by the Helmholtz-Society Portfolio grant "Technologie und Medizin - Multimodale Bildgebung zur Aufklärung des In-vivo-Verhaltens von polymeren Biomaterialien" by the Ligue Nationale Contre le Cancer and the Initiative d'Excellence grant from the University of Bordeaux.

\section{Disclosure}

The authors report no conflicts of interest in this work.

\section{References}

1. Wang L, Yang PP, Zhao XX, Wang H. Self-assembled nanomaterials for photoacoustic imaging. Nanoscale. 2016;8:2488-2509.

2. James ML, Gambhir SS. A molecular imaging primer: modalities, imaging agents, and applications. Physiol Rev. 2012;92:897-965.

3. Aldrich MB, Marshall MV, Sevick-Muraca EM, et al. Seeing it through: translational validation of new medical imaging modalities. Biomed Opt Express. 2012;3:764-776.

4. Beziere N, Lozano N, Nunes A, et al. Dynamic imaging of PEGylated indocyanine green (ICG) liposomes within the tumor microenvironment using multi-spectral optoacoustic tomography (MSOT). Biomaterials. 2015;37:415-424.

5. Luke GP, Yeager D, Emelianov SY. Biomedical applications of photoacoustic imaging with exogenous contrast agents. Ann Biomed Eng. 2012; 40:422-437.

6. Mallidi S, Luke GP, Emelianov S. Photoacoustic imaging in cancer detection, diagnosis, and treatment guidance. Trends Biotechnol. 2011;29: 213-221.

7. Lozano N, Al-Ahmady ZS, Beziere NS, Ntziachristos V, Kostarelos K. Monoclonal antibody-targeted PEGylated liposome-ICG encapsulating doxorubicin as a potential theranostic agent. Int J Pharm. 2015;482:2-10.
8. Wilson KE, Wang TY, Willmann JK. Acoustic and photoacoustic molecular imaging of cancer. J Nucl Med. 2013;54:1851-1854.

9. Porcu EP, Salis A, Gavini E, Rassu G, Maestri M, Giunchedi P. Indocyanine green delivery systems for tumour detection and treatments. Biotechnol Adv. 2016;34:768-789.

10. Hannah A, Luke G, Wilson K, Homan K, Emelianov S. Indocyanine green-loaded photoacoustic nanodroplets: dual contrast nanoconstructs for enhanced photoacoustic and ultrasound imaging. ACS Nano. 2014;8: $250-259$.

11. Song W, Tang Z, Zhang D, Burton N, Driessen W, Chen X. Comprehensive studies of pharmacokinetics and biodistribution of indocyanine green and liposomal indocyanine green by multispectral optoacoustic tomography. RSC Adv. 2015;5:3807-3813.

12. Alander JT, Kaartinen I, Laakso A, et al. A review of indocyanine green fluorescent imaging in surgery. Int J Biomed Imaging. 2012;2012: 940585.

13. Desmettre T, Devoisselle JM, Mordon S. Fluorescence properties and metabolic features of indocyanine green (ICG) as related to angiography. Surv Ophthalmol. 2000;45:15-27.

14. Shemesh CS, Moshkelani D, Zhang H. Thermosensitive liposome formulated indocyanine green for near-infrared triggered photodynamic therapy: in vivo evaluation for triple-negative breast cancer. Pharm Res. 2015;32:1604-1614.

15. Bahmani B, Lytle CY, Walker AM, Gupta S, Vullev VI, Anvari B. Effects of nanoencapsulation and PEGylation on biodistribution of indocyanine green in healthy mice: quantitative fluorescence imaging and analysis of organs. Int J Nanomedicine. 2013;8:1609-1620.

16. Kraft JC, Ho RJ. Interactions of indocyanine green and lipid in enhancing near-infrared fluorescence properties: the basis for near-infrared imaging in vivo. Biochemistry. 2014;53:1275-1283.

17. Mertens ME, Frese J, Bölükbas DA, et al. FMN-coated fluorescent USPIO for cell labeling and non-invasive MR imaging in tissue engineering. Theranostics. 2014;4:1002-1013.

18. Jayapaul J, Hodenius M, Arns S, et al. FMN-coated fluorescent iron oxide nanoparticles for RCP-mediated targeting and labeling of metabolically active cancer and endothelial cells. Biomaterials. 2011;32: 5863-5871.

19. Jayapaul J, Arns S, Bunker M, et al. In vivo evaluation of riboflavin receptor targeted fluorescent USPIO in mice with prostate cancer xenografts. Nano Res. 2016;9:1319-1333.

20. Beztsinna N, Solé M, Taib N, Bestel I. Bioengineered riboflavin in nanotechnology. Biomaterials. 2016;80:121-133.

21. Marlin F, Simon P, Bonneau S, et al. Flavin conjugates for delivery of peptide nucleic acids. Chembiochem. 2012;13:2593-2598.

22. Thomas TP, Choi SK, Li MH, Kotlyar A, Baker JR. Design of riboflavinpresenting PAMAM dendrimers as a new nanoplatform for cancertargeted delivery. Bioorg Med Chem Lett. 2010;20(17):5191-5194.

23. Massey V. The chemical and biological versatility of riboflavin. Biochem Soc Trans. 2000;28:283-296.

24. Johnson T, Ouhtit A, Gaur R, et al. Biochemical characterization of riboflavin carrier protein (RCP) in prostate cancer. Front Biosci (Landmark Ed). 2009;14:3634-3640.

25. Karande AA, Sridhar L, Gopinath KS, Adiga PR. Riboflavin carrier protein: a serum and tissue marker for breast carcinoma. Int $J$ Cancer. 2001;95:277-281.

26. Rao PN, Crippin J, Levine E, et al. Elevation of serum riboflavin carrier protein in hepatocellular carcinoma. Hepatol Res. 2006;35:83-87.

27. Jayapaul J, Arns S, Lederle W, et al. Riboflavin carrier protein-targeted fluorescent USPIO for the assessment of vascular metabolism in tumors. Biomaterials. 2012;33:8822-8829.

28. Beztsinna N, Tsvetkova Y, Bartneck M, Lammers T, Kiessling F, Bestel I. Amphiphilic phospholipid-based riboflavin derivatives for tumor targeting nanomedicines. Bioconjug Chem. 2016;27:2048-2061.

29. Torchilin V, Weissig V. Liposomes: A Practical Approach. Oxford: Oxford University Press; 2003.

30. Chang HI, Yeh MK. Clinical development of liposome-based drugs: formulation, characterization, and therapeutic efficacy. Int J Nanomedicine. 2012;7:49-60. 
31. Immordino ML, Dosio F, Cattel L. Stealth liposomes: review of the basic science, rationale, and clinical applications, existing and potential. Int J Nanomedicine. 2006;1:297-315.

32. McGhee W, inventor; Mallinckrodt Llc. Liposome oxaliplatin compositions for cancer therapy. WO2014159760 A1. 2014 Oct 2.

33. Hadjidemetriou M, Al-Ahmady ZS, Mazza M, Collins RF, Dawson KA, Kostarelos K. In vivo biomolecule corona around blood-circulating, clinically-used and antibody-targeted lipid bilayer nanoscale vesicles. ACS Nano. 2015;9(8):8142-8156.

34. Lammers T, Hennink WE, Storm G. Tumour-targeted nanomedicines: principles and practice. Br J Cancer. 2008;99:392-397.

35. Hama S, Itakura $S$, Nakai $M$, et al. Overcoming the polyethylene glycol dilemma via pathological environment-sensitive change of the surface property of nanoparticles for cellular entry. J Control Release. 2015;206:67-74.
36. Repenko T, Fokong S, De Laporte L, et al. Water-soluble dopaminebased polymers for photoacoustic imaging. Chem Commun. 2015;51: 6084-6087.

37. Morton CL, Houghton PJ. Establishment of human tumor xenografts in immunodeficient mice. Nat Protoc. 2007;2:247-250.

38. Oh JT, Li ML, Zhang HF, Maslov K, Stoica G, Wang LV. Threedimensional imaging of skin melanoma in vivo by dual-wavelength photoacoustic microscopy. J Biomed Opt. 2006;11:34032.

39. Kunjachan S, Pola R, Gremse F, et al. Passive versus active tumor targeting using RGD-and NGR-modified polymeric nanomedicines. Nano Lett. 2014;14:972-981.

40. Luke GP, Nam SY, Emelianov SY. Optical wavelength selection for improved spectroscopic photoacoustic imaging. Photoacoustics. 2013; $1: 36-42$. 


\section{Supplementary materials}

A

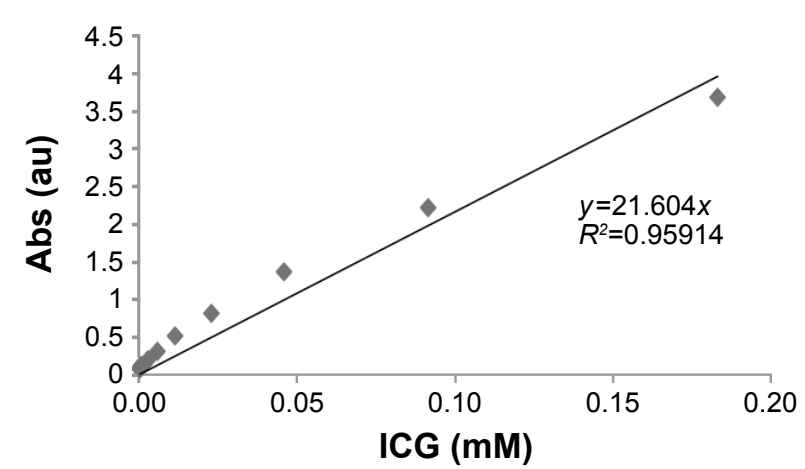

C

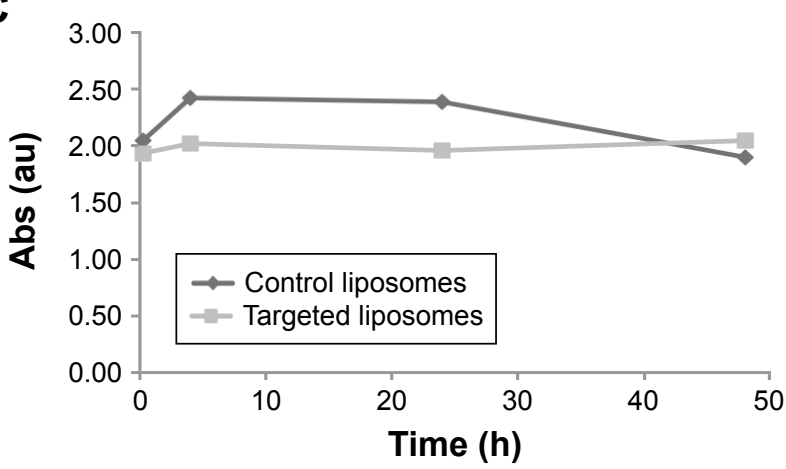

B

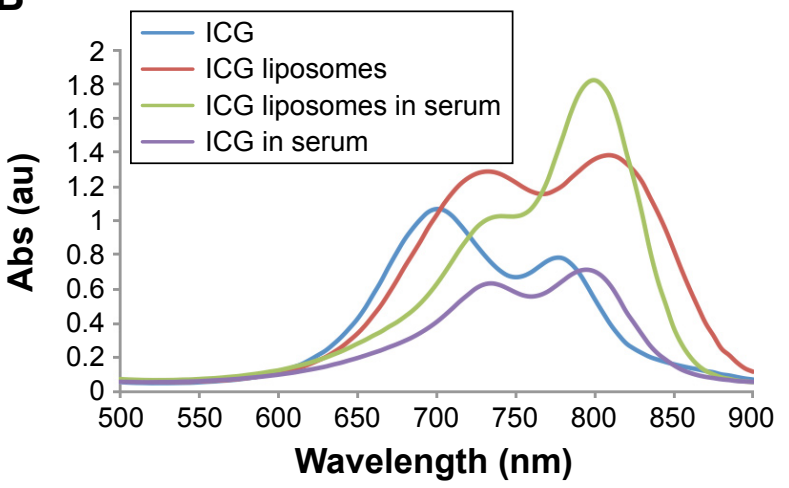

D

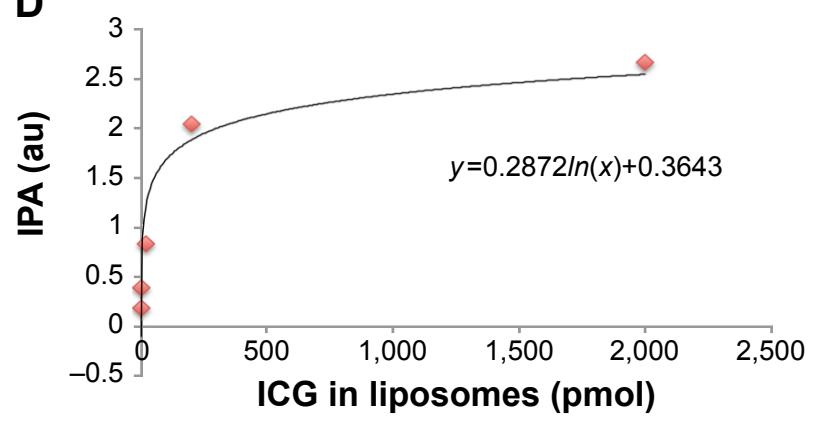

Figure SI (A) Calibration curve for ICG in buffer, absorbance measured at $780 \mathrm{~nm}$. (B) ICG absorbance in buffer (blue) or $50 \%$ FBS (purple), encapsulated in liposomes dispersed in buffer (red) or 50\% FBS (green). (C) ICG absorbance signal from liposomes dispersed in $50 \%$ FBS at $37^{\circ} \mathrm{C}$ over time. (D) Photoacoustic signal intensity from ICG in liposomes measured in polymeric phantoms.

Abbreviations: ICG, indocyanine green; FBS, fetal bovine serum; Abs, absorbance; h, hours.

Table SI Characteristics of RhB-encapsulating liposomes

\begin{tabular}{|c|c|c|c|c|c|}
\hline Name & Lipids & Size $(\mathrm{nm})$ & PDI & Zeta potential (mV) & $\mathbf{R h B}(\mu \mathbf{M})$ \\
\hline Control liposomes RhB & $\begin{array}{l}\text { DPPC:chol:DSPE-PEG-RF } \\
65: 30: 5\end{array}$ & 86 & 0.257 & -8 & 0.48 \\
\hline Targeted liposomes RhB & $\begin{array}{l}\text { DPPC:chol:DSPE-PEG-RF } \\
65: 30: 5\end{array}$ & 91 & 0.267 & -21 & 0.48 \\
\hline Competition & $\begin{array}{l}\text { DPPC:chol:DSPE-PEG-RF } \\
65: 30: 5\end{array}$ & 154 & 0.084 & -21 & - \\
\hline
\end{tabular}

Abbreviations: PDI, polydispersity index; RhB, rhodamine B.
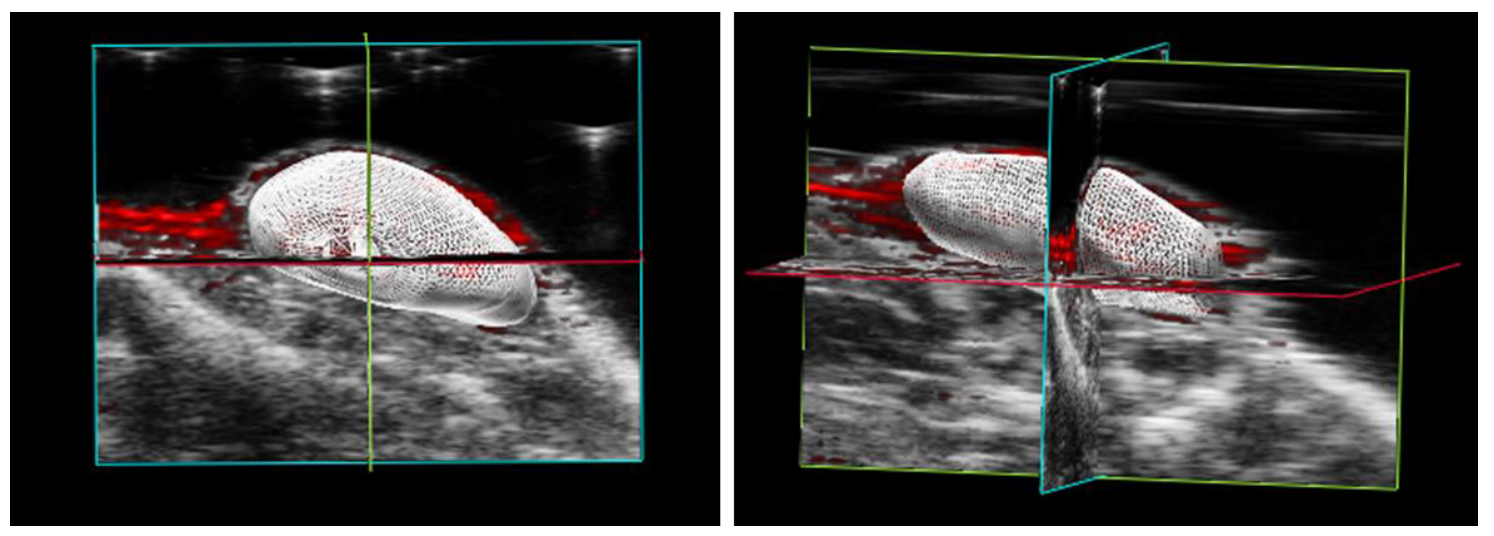

Figure S2 Example of the 3D tumor volume reconstruction: front (left) and side (right) view. PS\% is assigned to the reconstructed 3D volume. Abbreviations: PS\%, photoacoustic signal; 3D, three-dimensional. 


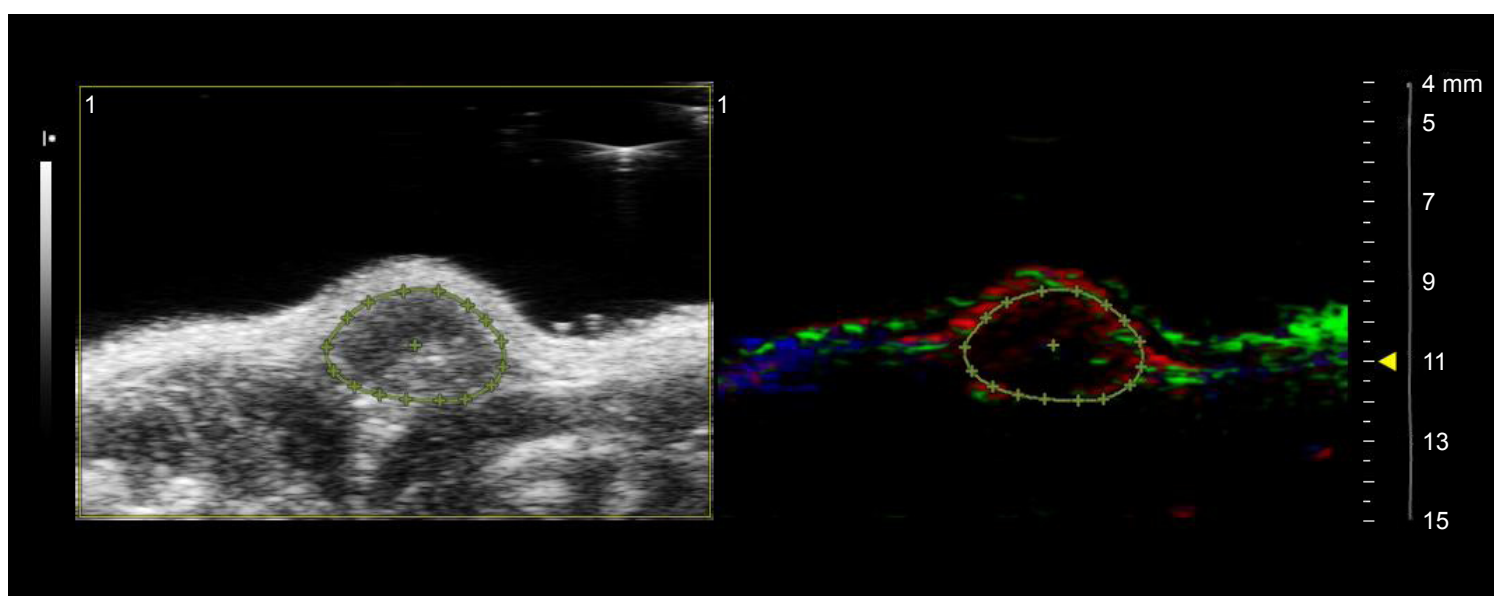

Figure S3 Example of the region of interest measurement in the unmixed two-dimensional tumor slice.

Notes: Ultrasound signal - black and white; indocyanine green signal from liposomes - green; OxyHemo - red; and deOxyHemo - blue.

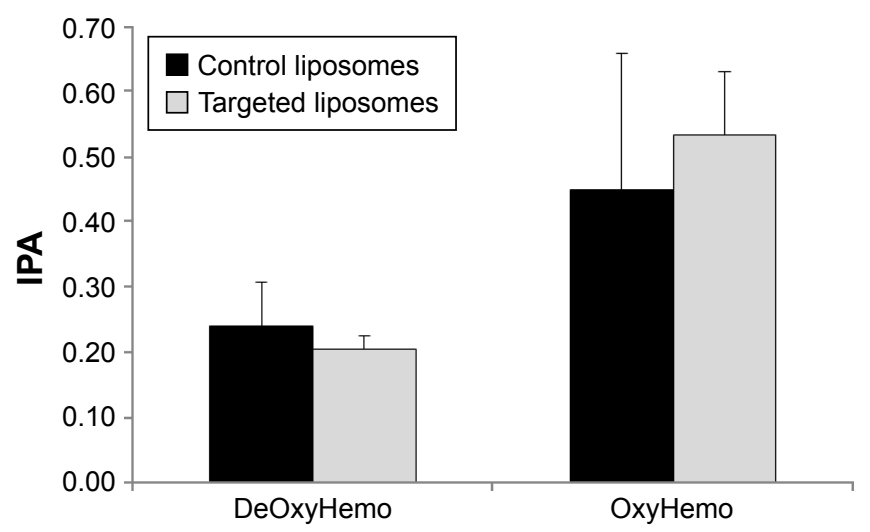

Figure S4 Quantification of unmixed IPA of oxygenated and deoxygenated hemoglobin from two-dimensional tumor slices in control and targeted groups. Note: Data are presented as mean \pm standard deviation from $n=2$ mice/group.

Abbreviations: IPA, PA average intensity; PA, photoacoustic.

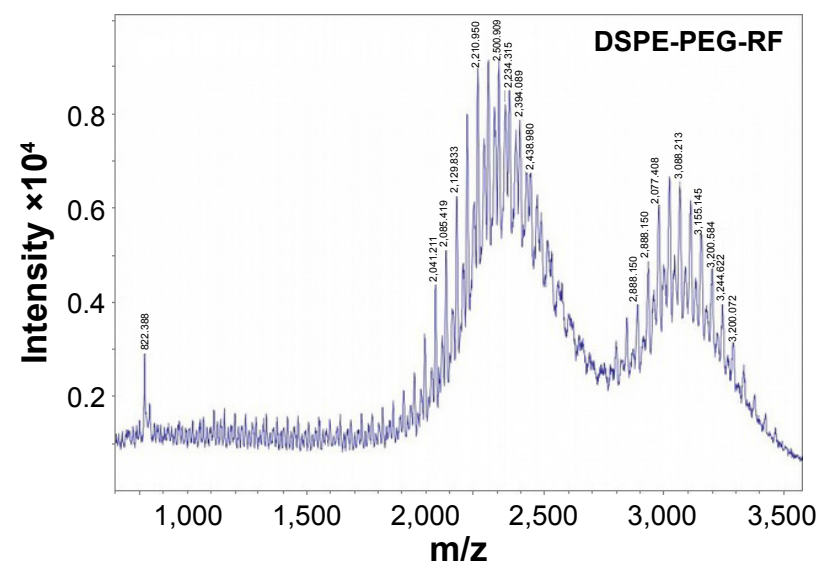

Figure S5 MALDI-TOF-TOF (Bruker Daltonics GmbH) spectrum of DSPE-PEG-RF. 


\section{Size distribution by intensity}

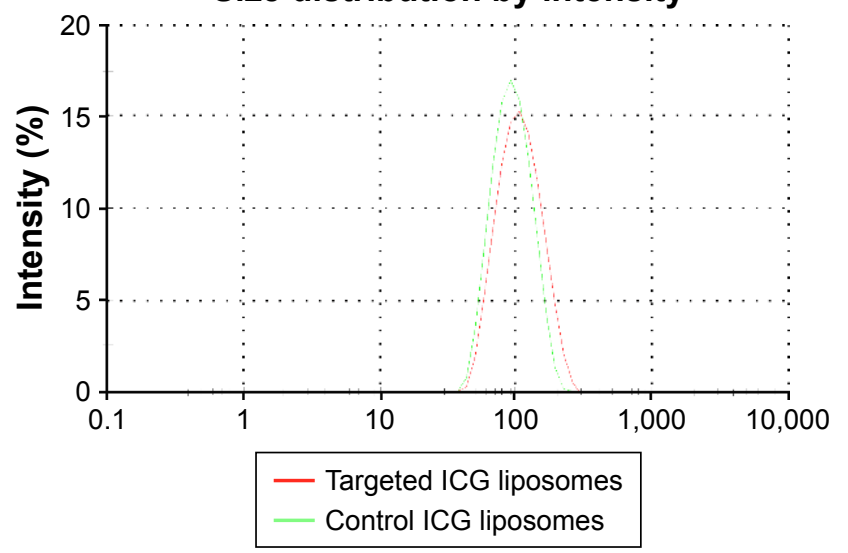

Figure S6 Size distribution by intensity of control (green) and targeted (red) ICG-encapsulating liposomes.

Abbreviation: ICG, indocyanine green.

\section{Publish your work in this journal}

The International Journal of Nanomedicine is an international, peerreviewed journal focusing on the application of nanotechnology in diagnostics, therapeutics, and drug delivery systems throughout the biomedical field. This journal is indexed on PubMed Central, MedLine, CAS, SciSearch ${ }^{\circledR}$, Current Contents ${ }^{\circledR} /$ Clinical Medicine,
Journal Citation Reports/Science Edition, EMBase, Scopus and the Elsevier Bibliographic databases. The manuscript management system is completely online and includes a very quick and fair peer-review system, which is all easy to use. Visit http://www.dovepress.com/ testimonials.php to read real quotes from published authors.

Submit your manuscript here: http://www.dovepress.com/international-journal-of-nanomedicine-journal 